\title{
Ring current development during storm main phase
}

\author{
Mei-Ching Fok \\ Universities Space Research Association, Space Sciences Laboratory, NASA Marshall Space Flight \\ Center, Huntsville, Alabama
}

Thomas E. Moore

Space Sciences Laboratory, NASA Marshall Space Flight Center, Huntsville, Alabama

Marian E. Greenspan

Department of Physics, University of Maryland, College Park, Maryland

\begin{abstract}
The development of the ring current ions in the inner magnetosphere during the main phase of a magnetic storm is studied. The temporal and spatial evolution of the ion phase space densities in a dipole field are calculated using a three dimensional ring current model, considering charge exchange and Coulomb losses along drift paths. The simulation starts with a quiet time distribution. The model is tested by comparing calculated ion fluxes with Active Magnetospheric Particle Tracer Explorers/CCE measurement during the storm main phase on May 2, 1986. Most of the calculated omnidirectional fluxes are in good agreement with the data except on the dayside inner edge $(L<2.5)$ of the ring current, where the ion fluxes are underestimated. The model also reproduces the measured pitch angle distributions of ions with energies below $10 \mathrm{keV}$. At higher energy, an additional diffusion in pitch angle is necessary in order to fit the data. The role of the induced electric field on the ring current dynamics is also examined by simulating a series of substorm activities represented by stretching and collapsing the magnetic field lines. In response to the impulsively changing fields, the calculated ion energy content fluctuates about a mean value that grows steadily with the enhanced quiescent field.
\end{abstract}

\section{Introduction}

During the main phase of a magnetic storm, energetic plasmas are injected on the nightside from a boundary near the geosynchronous orbit. In response to the convection electric field, these particles drift inward and are trapped by the geomagnetic field and form the storm time ring current. The development of the ring current during the main phase of a magnetic storm has been studied. Wolf et al. [1982] applied the Rice convection model [Harel et al., 1981] to study the early main phase of the storm of July 29,1977 . They found the magnetospheric convection, including effects of shielding, was sufficient to inject enough plasma sheet plasma deep into the magnetosphere to form a storm time ring current. Chen et al. [1994] used a guiding center simulation to model the main phase of storms as a sequence of substorm-associated enhancements in the convection electric field. They found that for storms with a main phase of about 3 hours the ring current enhancements are mainly associated with ions injected from open trajectories to closed ones. The ring current is augmented also by diffusive transport of higher-energy ions $(E \geq 160$ $\mathrm{keV}$ ) for storms having longer main phases ( $\geq 6$ hours). They also found that transport alone would not account for the entire decrease in Dst typical for a major storm but an increased boundary value of the phase space density was necessary.

Copyright 1996 by the American Geophysical Union.

Paper number 96JA01274.

0148-0227/96/96JA-01274\$09.00
In this paper, some problems of convection, collisional loss, and pitch angle diffusion effects on the ring current development during the storm main phase are addressed. The main phase of the magnetic storm on May 2, 1986, is simulated using a three-dimensional ring current model described by Fok et al. [1993, 1995a]. The model assumes a dipole magnetic field and the convection electric field of Volland-Stern, parameterized by the $K p$ index [Volland, 1973; Stern, 1975; Maynard and Chen, 1975], is employed. The special features of the model are the consideration of ions with arbitrary pitch angle distribution and the inclusion of Coulomb interactions with the thermal plasma in the plasmasphere. This model is developed not only for studying the ring current dynamics but it is also a useful tool to examine the interactions between the ring current and the background neutral and plasma populations [Fok et al., 1993, 1995b]. The description of the model and the results in simulating the recovery phases of a moderate and a major storm are described briefly in the next section. In the following, only the evolution of $\mathrm{H}^{+}$ions during the development of the storm is discussed. The simulation starts with a quiet time distribution [Sheldon and Hamilton, 1993] at $L$ shells between 2 and 6.5 . The nightside energy spectra of $\mathrm{H}^{+}$fluxes measured by the Active Magnetospheric Particle Tracer Explorers (AMPTE)/CCE at $L \sim 6.75$ at every 16 hours serve as the model boundary conditions. The measured omnidirectional fluxes and pitch angle distributions (PADs) inside the injection boundary are compared with model predictions.

The convection electric field model is a crucial factor in determining the particle drifts and thus the global ring current 
dynamics. Usually, the convection field can be separated into an electrostatic component and a substorm-induced component. In this study, the different roles of the two components of the electric field on the ring current development will be examined. The ring current model will be run with an induced electric field in addition to the VollandStern-type convection, and the results will be compared with those from calculations in which the induced field is not included. The algorithm we use to construct the time-dependent induced field is different from those presented in previous works, and it will be described in section 6 . In the following, average flux means the average differential intensity (in $\mathrm{s}^{-1} \mathrm{~cm}^{-2} \mathrm{sr}^{-1} \mathrm{keV}^{-1}$ ) over pitch angles, except where another definition is specifically given.

\section{The Model}

The 3-D ring current model solves the following bounceaveraged kinetic equation of the phase space density of the ring current ion species, considering charge exchange and Coulomb losses along the drift path [Fok et al., 1993, 1995a]:

$$
\begin{aligned}
\frac{\partial \bar{f}_{s}}{\partial t}+\left\langle\dot{R}_{\mathrm{o}}\right\rangle \frac{\partial \bar{f}_{s}}{\partial R_{\mathrm{o}}}+\langle\dot{\phi}\rangle \frac{\partial \bar{f}_{s}}{\partial \phi}= & -v \sigma_{s}\left\langle n_{H}\right\rangle \bar{f}_{s} \\
& +\frac{1}{M^{1 / 2}} \frac{\partial}{\partial M}\left(\langle\dot{M}\rangle M^{1 / 2} \bar{f}_{s}\right)
\end{aligned}
$$

where $\bar{f}_{s}\left(R_{0}, \phi, M, K\right)$ is the average phase space distribution function of species $s$ along the field line between mirror points, $R_{0}$ is the radial distance at the equator, $\phi$ is the magnetic local time, $M$ is the magnetic moment, $v$ is velocity, $\sigma_{s}$ is the cross section for charge exchange of species $s$ with the neutral hydrogen, $n_{H}$ is the hydrogen density, and $\dot{M}$ is the rate of change of $M$ due to Coulomb interactions with the thermal plasmas. $K$ is defined as [Mcllwain, 1966],

$$
K \equiv J /\left(8 m_{s} M\right)^{1 / 2}
$$

and it is an invariant in the case of zero parallel electric field [Roederer, 1970]. The notation $\langle x\rangle$ is the bounce-averaged value of $x$. Since we are considering particles with bounce periods much shorter than the decay lifetimes, $f_{s}$ is assumed to be constant along the field line, and thus $\bar{f}_{s}$ can be replaced by the distribution function at the equator. The temporal evolution of the energy and pitch angle dependence on the ion distribution in a 3-D spatial space can be inferred from the information on the equator. In solving the kinetic equation (1), the time-splitting method is employed. At each fractional time step, only one process is considered [Fok et al., 1993, 1995a].

The ring current model has been used to model the recovery phases of a moderate storm and a major storm. The model ion differential fluxes in general agree well with the observations from AMPTE/CCE, except the calculated $\mathrm{H}^{+}$fluxes at energy less than $100 \mathrm{keV}$ always exceed those of measurement [Fok et al., 1995a]. However, our model successfully reproduces many observed features of storm recovery, for example, drift holes on the dayside at energies of $3-20 \mathrm{keV}$ and separation of ions as a function of energy due to differences in drift velocities and charge exchange lifetimes. The 3-D model also predicts the buildup of pitch angle anisotropy observed during storm recovery.

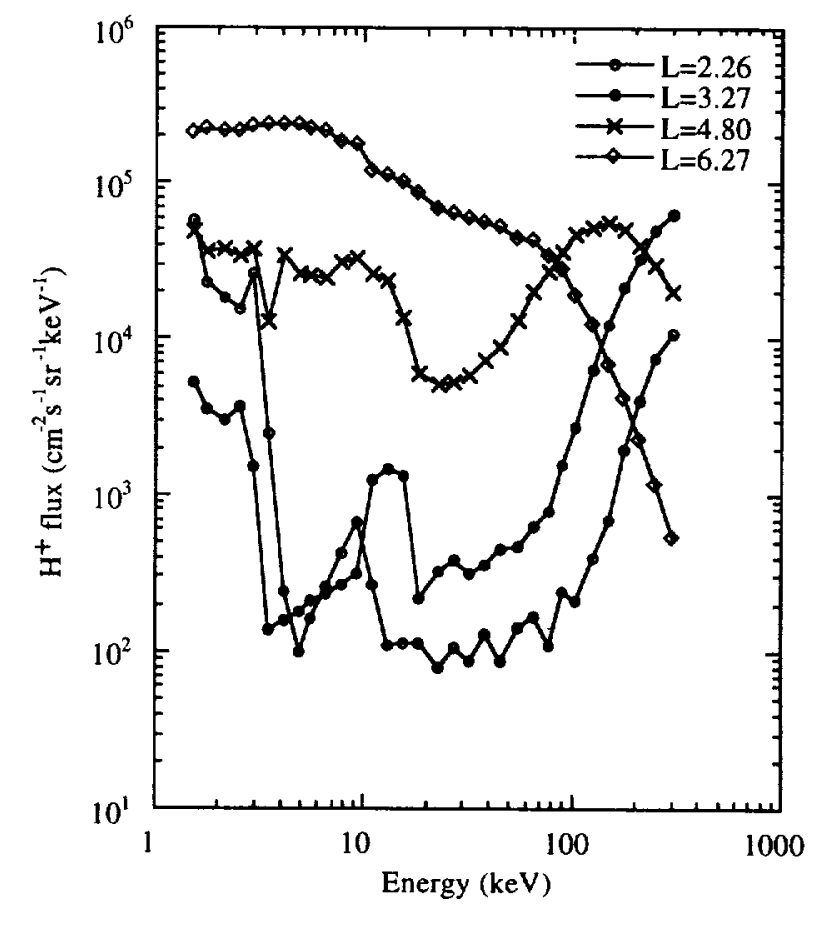

Figure 1. Quiet time equatorial $\mathrm{H}^{+}$fluxes at representative $L$ shells [Sheldon and Hamilton, 1993].

\section{Initial Conditions: Quiet Time Conditions}

We start the simulation with quiet time conditions. The ion composition compiled by Sheldon and Hamilton [1993] during the quietest days in 1985-1987, seen by the AMPTE/CCE/ charge-energy-mass (CHEM) instrument in near-equatorial orbit at $L=2-9 R_{E}$, is used as initial distribution before storm onset. This data set provides average differential ion fluxes in an energy range $1-300 \mathrm{keV}$. The initial energy spectra of average $\mathrm{H}^{+}$fluxes at some representative $L$ shells are plotted in Figure 1. This quiet time distribution is assumed to be local time symmetric, although Sheldon and Hamilton [1993] found a small dawn-dusk distortion caused by the cross-tail electric field.

The quiet time PAD of ring current ions is estimated by the charge exchange cross sections, assuming that the PAD is mainly shaped by the charge exchange loss. The initial value of the exponential index $n$ of the pitch angle fit $(j(y)=$ $j_{\mathrm{o}}\left(1+A y^{n}\right), y=$ sine of the equatorial pitch angle) of $\mathrm{H}^{+}$is given as follows, in light of previous modeling results at late recovery [Fok et al., 1995a]:

$$
n=73.75 \frac{\left(\sigma_{s} \sqrt{E}\right)^{0.613}}{L^{2.74}}
$$

where $\sigma_{s}$ is in $10^{-19} \mathrm{~m}^{2}$ and $E$ is in $\mathrm{keV}$. The value of $n$ increases with decreasing $L$ and reaches a value greater than 6 near the inner edge of the ring current for energies below tens of $\mathrm{keV}$. A relatively isotropic distribution of high-energy (>70 keV) $\mathrm{H}^{+}$is obtained, as a result of its large charge exchange lifetime. However, using data from the Explorer 45 satellite, Williams and Lyons [1974] found rounded PADs (peak at $\alpha=\pi / 2$ ) of high energy $\mathrm{H}^{+}$for altitudes in the 


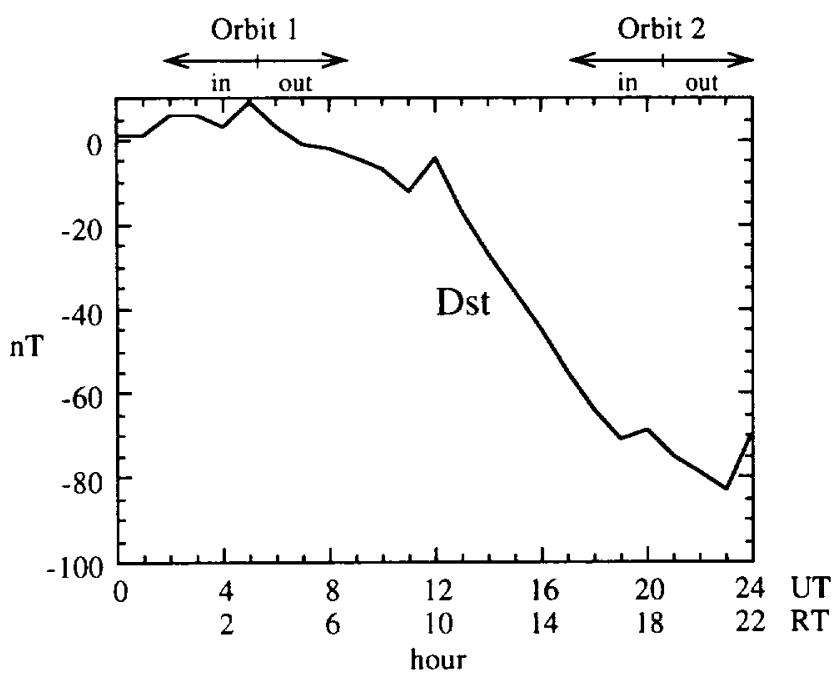

CCE Orbit

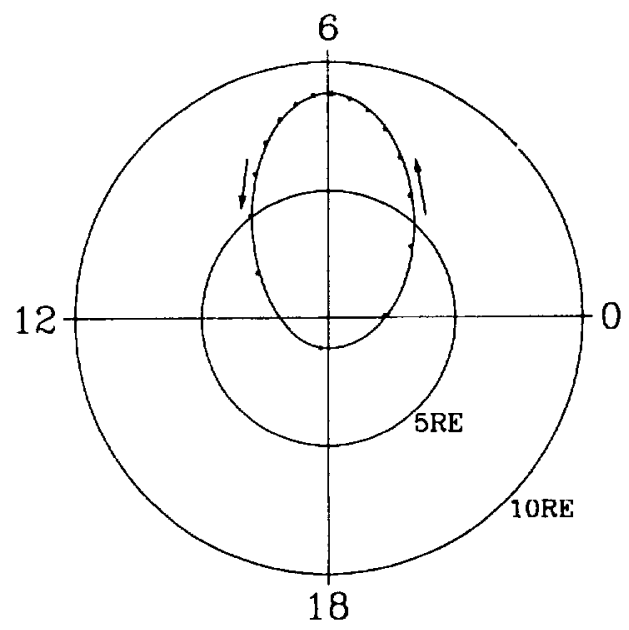

Figure 2. (top) Dst index and (bottom) the CCE orbit on May 2, 1986. The times during the two orbit passes in the ring current region are 0150-0840 UT for Orbit 1 and 1720-2400 UT for Orbit 2. Marks on the orbit are at 1-hour intervals.

plasmasphere region. They interpreted these PADs as a consequence of pitch angle diffusion due to the amplification of ion cyclotron waves when the hot ring current plasma interacts with the cold plasmaspheric plasma. In section 5 , we will evaluate the effect of wave-particle interactions on the $\mathrm{H}^{+}$ PADs, especially at high energies.

The development during the main phase of a magnetic storm, similar to that on May 2, 1986, is studied. The Dst index and the CCE orbit on May 2, 1986, are shown in Figure 2. In the following, we only consider $\mathrm{H}^{+}$, which is the dominant ion species for this storm. The simulation starts at 0200 UT (run time, RT $=0$ ), when the geomagnetic conditions are quiet. An empty loss cone distribution is assumed at RT = 0 . During the simulation, an additional loss term with a lifetime of half of the bounce period is applied to ions in the loss cone, which is defined at $800 \mathrm{~km}$. The measured AMPTE/CCE $\mathrm{H}^{+}$flux as a function of pitch angle at $L \sim 6.75$, LT $\sim 4$ hours, at RT $=6$ (Orbit 1 ) and 22 (Orbit 2) hours are used to update the nightside boundary condition during the storm main phase.

\section{Energy Spectra During Storm Main Phase}

The development of ring current $\mathrm{H}^{+}$during the storm main phase on May 2, 1986, is simulated with the initial and boundary conditions specified in the last section. The offset in the convection potential is assumed to be 2 hours, making the convection paths symmetric about the meridian of $0800-2000$ LT [Kistler et al., 1989]. The simulated $\mathrm{H}^{+}$fluxes are compared with the CCE measurements during Orbit 2. Figure 3 shows the comparison during the inbound (Figures $3 \mathrm{a}-3 \mathrm{c}$ ) and outbound (Figures 3d-3f) pass at $L \sim 5.75,3.25$, and 2.25. There are minima in the dayside spectra at about $10 \mathrm{keV}$ seen by CHEM measurements. These dips are consistent features of the storm time ring current ions, as a result of slow drift velocity [Mcllwain, 1972; Fok et al., 1995a]. As shown in Figure 3, our model reproduces the "drift holes" at $L<4$. However, the simulation predicts a lack of low-energy (below tens of keV) ions at the inner edge of the ring current (Figure $3 \mathrm{c}$ ) due to significant charge exchange loss before particles drift from the nightside source region to the dayside observation point. Significant low-energy $\mathrm{H}^{+}$fluxes of $10^{5} \mathrm{~s}^{-1} \mathrm{~cm}^{-2} \mathrm{st}^{-1} \mathrm{keV}^{-1}$ are measured. These low-energy ions may be a result of direct injection from the ionosphere [Horwitz, 1982] or plasmasphere during the main phase. Also radial diffusion induced by electric and magnetic fluctuations could diffuse ions from the boundary of open and closed drift paths to lower $L$ shells. These transport mechanisms are not included in the present model. Radial diffusion is believed to be the major transport process for high-energy ( $E \geq 280 \mathrm{keV}$ at $L \sim 3$ ) ions [Chen et al., 1993]. However, the good agreement between the modeling fluxes and the measured fluxes at high energies shown in Figure 3 indicates that this diffusion process has only a small effect on redistributing energetic ions in radial distance.

The simulated $\mathrm{H}^{+}$fluxes on the nightside agree well with measurements (Figures 3d-3f). At $L \sim 5.75$, the ion flux is similar to a typical geosynchronous distribution, which is a double Maxwellian with peaks at a few $\mathrm{keV}$ and tens of $\mathrm{keV}$ [Borovsky et al., 1994]. At low $L$ shells (Figures 3d and 3e), the spectra show the overlapping of two ion populations: the freshly injected low-energy ions from the tail and the preexistent high-energy particles. In this case, the dips on the energy spectra at $\sim 80-100 \mathrm{keV}$ correspond to the transition energy between the open and closed drift paths.

The good agreement shown in Figure 3 between the simulated ion fluxes and measurements affirms the ability of our model to reproduce energy features of the storm time ring current. We next display the spatial distribution of these energy features in the average $\mathrm{H}^{+}$fluxes at the equator during the main phase of the storm. Fok et al. [1995a] used chromograms to display the global energy distribution of ion fluxes. A description of chromogram can be found in the work by Fok et al. [1995a] and is also given in the appendix. In Plate 1 , ion fluxes are divided into three energy ranges: 1-5, $5-40$, and $40-300 \mathrm{keV}$. They are represented by red, green, and blue (color wheels in Plate 1) in the chromogram, respectively. The geometric mean of the two boundaries for each energy range is also labeled on the color wheel. The gray 

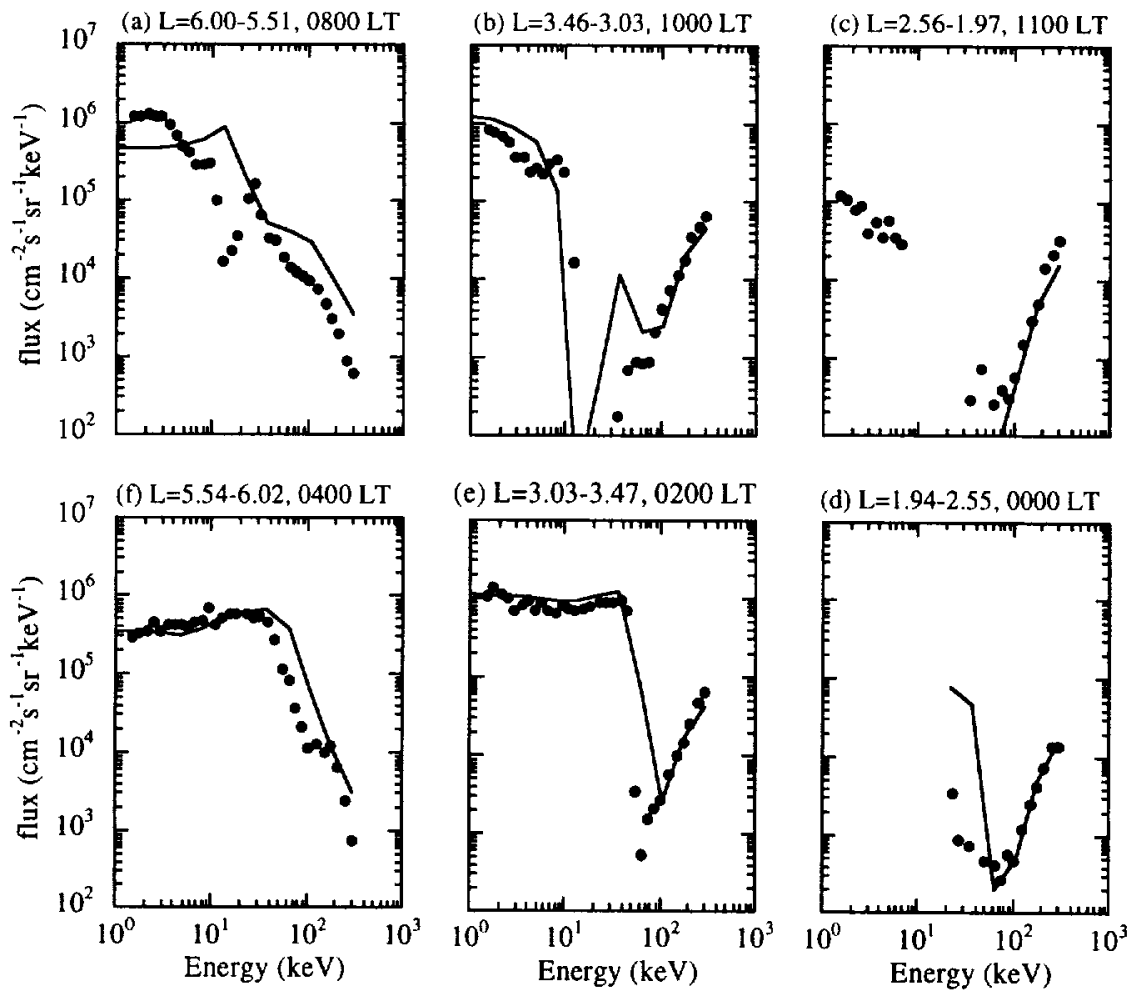

Figure 3. The comparison of calculated average $\mathrm{H}^{+}$fluxes (curves) with Active Magnetospheric Particle Tracer Explorers (AMPTE)/CCE measurements (circles) during Orbit 2 at selected locations.

scale bar is a guide to the interpretation of brightness, which represents the average flux intensity over the entire energy range. Contours of brightness are overlaid on the chromogram to distinguish brightness from color.

Plate 1 shows chromogram representations of $\mathrm{H}^{+}$average fluxes at the beginning, middle, and end of the storm main phase. The $K p$ value as a function of universal time on May 2, 1986 , and run time is given in the upper left panel. At RT $=1$ hour (Plate 1a), the $\mathrm{H}^{+}$flux is mostly a quiet time distribution (cf. Figure 1). High-energy $\mathrm{H}^{+}$dominates at $L<3$ (dark blue area). Low-energy fluxes increase with $L$ shells and are indicated by the magenta ring at $3<L<5$, where high- and low-energy ions coexist with a lack of medium-energy particles. An injection of ions is seen at the nightside geosynchronous region. The injections are mainly carried by ions of energies less than $40 \mathrm{keV}$ (yellow fringe). In response to the intensifying convection field, the freshly injected ions move earthward and result in a strong day-night energy asymmetry in flux level at RT $=9$ hours (Plate lb). The total ion flux is increasing during the main phase. It should be noted that the intensity range indicated by the gray scale bars increases for each successive panel in Plate 1. As shown in Plate $1 \mathrm{~b}$, less energetic ions corotate and reach the dayside through dawn (red area in the dawn-noon quadrant). Energetic ions drift westward and may move out of the model boundary in the noon-dusk quadrant (green-cyan area). The blue area near the inner edge of ring current is the preexisting high-energy ions, whose drift paths are closed so they cannot be transported from the tail through open drift paths. The green and blue areas form a boundary between open and closed drift paths for the low-energy $(<5 \mathrm{keV})$ ions. At RT $=21$ hours
(Plate $1 \mathrm{c}), K p$ reaches a value of $7^{+}$. Ions penetrate closely to the Earth on the nightside. The maximum ion flux is located at $L \sim 4.5$. The light yellow represents a flat energy distribution of ions in this region, with a slight peak at low energy. The ares forbidden to the convecting low-energy ions is compressed in response to the strong convection field.

\section{Pitch Angle Distribution}

The spin of the CCE spacecraft allows particle sampling at all pitch angles [Williams and Sugiura, 1985]. Figure 4 shows the measured differential fluxes (circles) in four energy ranges as a function of the equatorial pitch angle during Orbit 2. Data in the inbound (dayside) pass are shown except for measurements in the energy range of $17.9-28.2 \mathrm{keV}$, where data in the outbound (nightside) pass are plotted because of the low flux level on the dayside at these energies. At $L \sim 2.75$ (left panels), ion fluxes of all energies strongly peak at $90^{\circ}$ as a result of rapid charge exchange loss of particles with small pitch angles. The distributions are flatter as $L$ is increasing, where the neutral hydrogen density is low. Near geosynchronous orbit (right panels), peak fluxes of lowenergy $(<30 \mathrm{keV})$ ions are found at field-aligned pitch angles. This enhancement of field-aligned low-energy particles during storm injections has been observed by the ATS 6 geosynchronous satellite and has been suggested as a signature of the parallel energization mechanism [Mauk, 1986].

Simulated PADs plotted in solid curves are overlaid with the CCE measurements in Figure 4. Calculated fluxes are scaled by the measured fluxes near $90^{\circ}$ pitch angle in order to compare the shapes of the distributions. The calculated field-aligned 

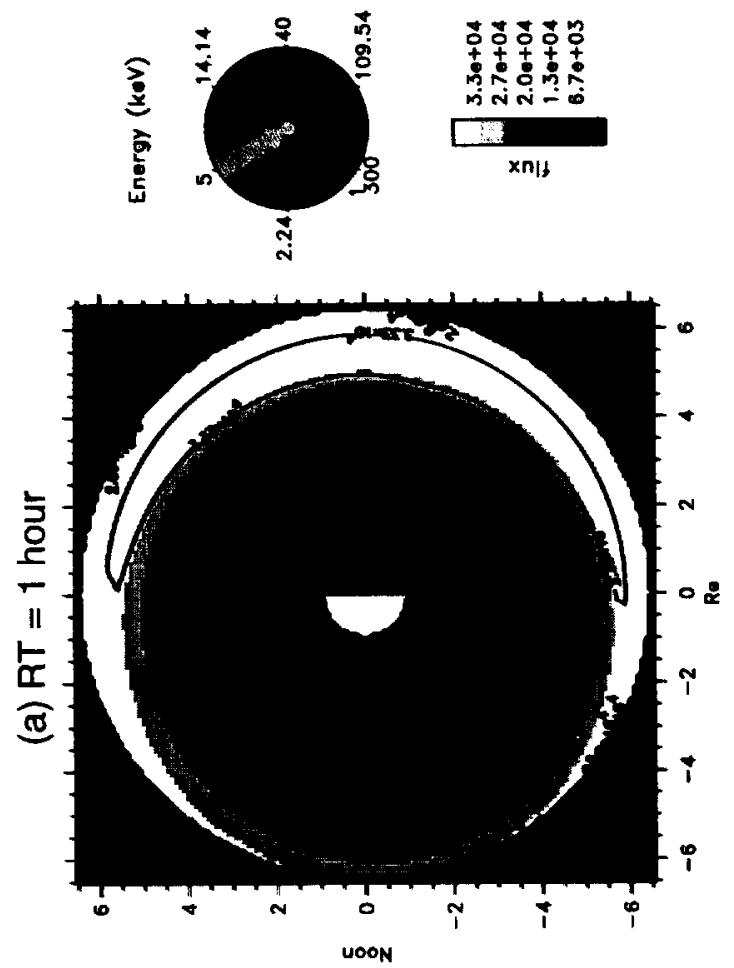

言言

$5 \mathrm{x}$

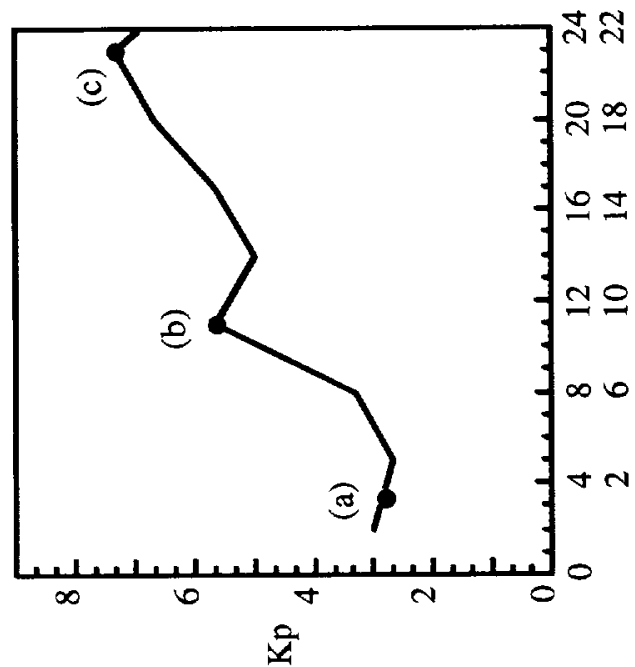

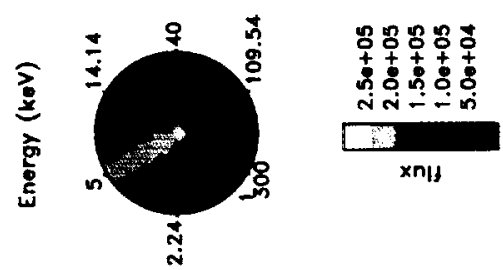
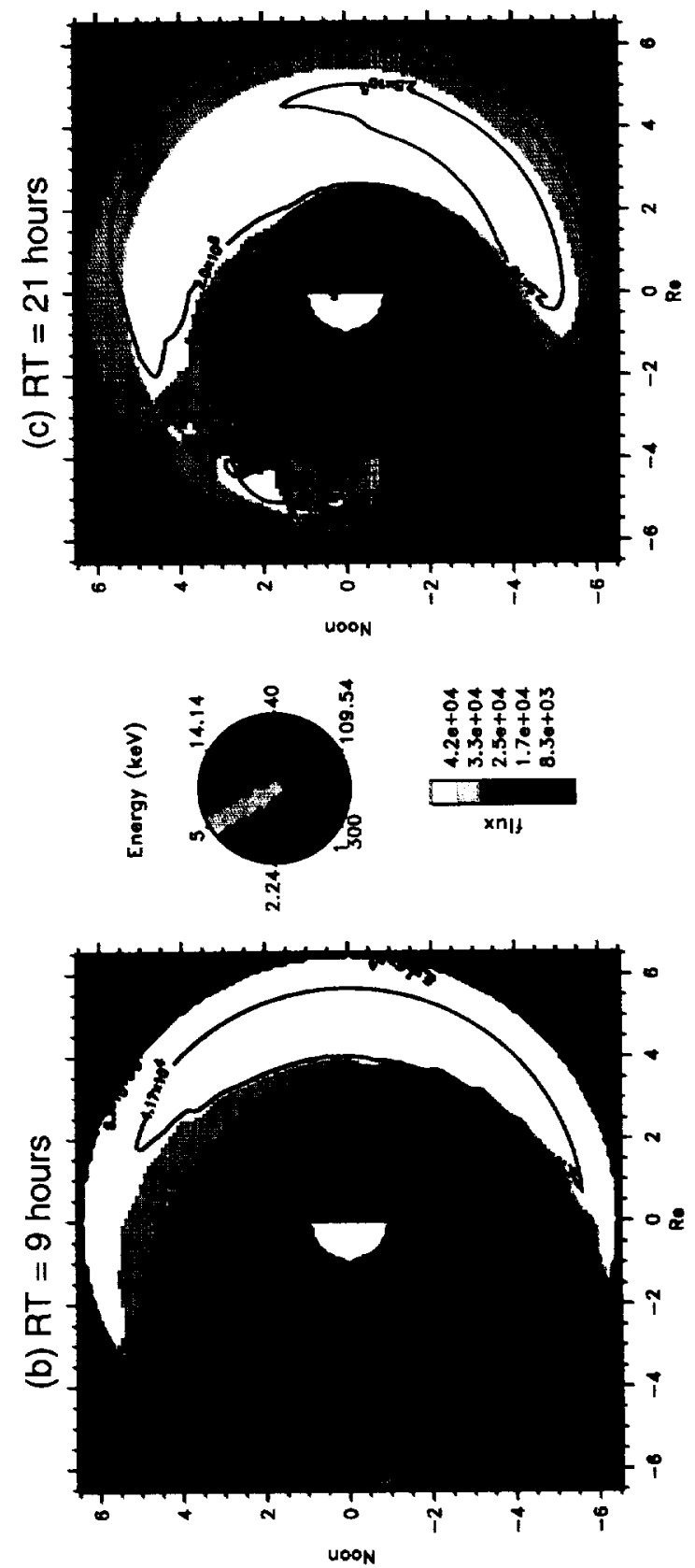

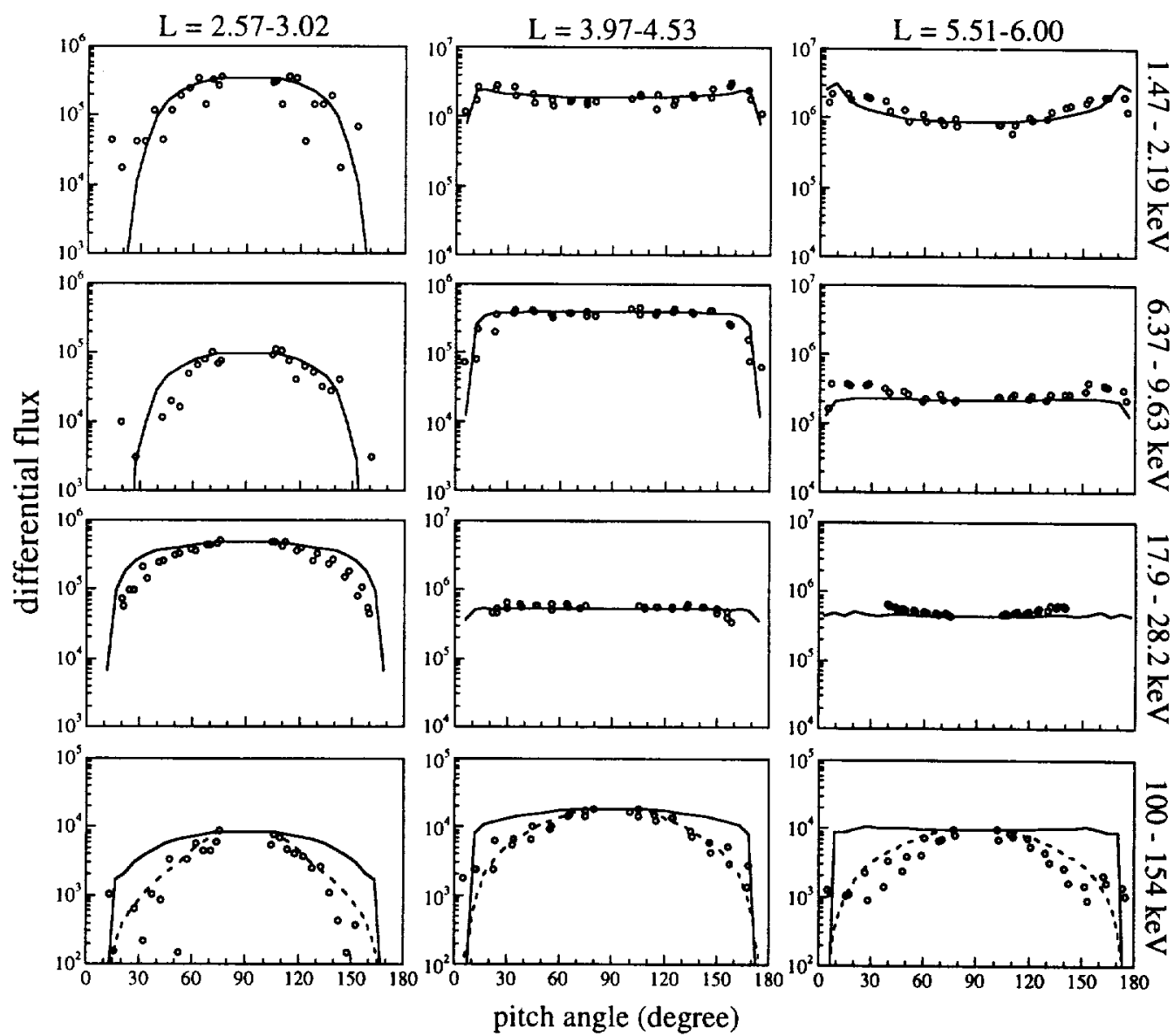

Figure 4. The comparison of calculated $\mathrm{H}^{+}$fluxes as a function of the equatorial pitch angle (curves) with AMPTE/CCE measurements (circles). Dashed curves in the bottom panels (100-154 $\mathrm{keV}$ ) represent distributions when an additional pitch angle diffusion, with $D_{\alpha \alpha}=5 \times 10^{-6} \mathrm{~s}^{-1}$, is considered.

distribution at 1.47-2.19 keV, at $L \sim 5.75$ (top right panel) results from the boundary PAD at $L=6.75$. It can be seen that our model agrees with data very well at low energies. This implies that PADs of low energy ions are mainly controlled by the drift motion and the charge exchange process, which are considered in our model. At high energies (bottom panels), the model predicts a flat distribution of trapped ions and a sharp drop-off near the loss cone. This predicted flat distribution on the dayside is a result of the isotropic boundary condition on the nightside. However, the data on the dayside show a gradual decrease in flux from the trap region to the loss cone. It seems that some diffusion processes in pitch angle, most likely due to wave-particle interactions, must take place to smooth out the distributions. Kennel and Petschek [1966] showed that for ion energies greater than $E_{c}=B^{2} / 2 \mu_{0} n_{b}$, where $n_{b}$ is the cold plasma density, the interaction with cyclotron waves leads to pitch angle diffusion. At $L=5$, assuming $n_{b}=10 \mathrm{~cm}^{-3}, E_{c} \sim 15$ $\mathrm{keV}$ is obtained. This estimate supports the argument that interactions with ion cyclotron waves play a more important role in determining PAD for ions of energies at or above tens of $\mathrm{keV}$ than ions of lower energies.

In order to estimate the wave amplitude that could account for the measured PAD, an additional term of pitch angle diffusion is added in the kinetic equation (1), with the diffusion coefficient chosen such that the calculated PAD would best fit the data. Consider a pure pitch angle diffusion in the energypitch angle space $(E, \alpha)$ :

$$
\frac{\partial f_{s}}{\partial t}=\frac{1}{\sin \alpha} \frac{\partial}{\partial \alpha}\left(\sin \alpha D_{\alpha \alpha} \frac{\partial f_{s}}{\partial \alpha}\right)
$$

Carrying out the transformation from $(E, \alpha)$ to $(E, K)$, we have

$$
\frac{\partial f_{s}}{\partial t}=2 R_{\mathrm{o}} \cos \alpha T(y) \frac{\partial}{\partial K}\left(D_{\alpha \alpha} \frac{2 B_{\mathrm{o}} R_{\mathrm{o}} T(y)}{y^{2}} \frac{\cos \alpha}{\sin ^{2} \alpha} \frac{\partial f_{s}}{\partial K}\right)
$$

where $y$ is the sine of the equatorial pitch angle and $T(y)$ is defined as [Davidson, 1976]

$$
T(y) \equiv \frac{1}{R_{\mathrm{o}}} \int_{0}^{S_{m}} \frac{d S}{\cos \alpha}
$$

Bounce-averaging (5) gives

$$
\frac{\partial \bar{f}_{s}}{\partial t}=\frac{\partial}{\partial K}\left(\left\langle D_{K K}\right\rangle \frac{\partial \bar{f}_{s}}{\partial K}\right)
$$

where 


$$
\left\langle D_{K K}\right\rangle=B_{\mathrm{o}}\left(\frac{2 R_{\mathrm{o}} T(y)}{y}\right)^{2}\left\langle\frac{D_{\alpha \alpha}}{\tan ^{2} \alpha}\right\rangle
$$

Therefore $\left\langle D_{K K}\right\rangle$ can be calculated for a given $D_{\alpha \alpha}$. Since the phase space density in our model (1) is expressed in terms of $(M, K)$, at each time step, before the diffusion term of $(7)$ is solved, $\bar{f}_{s}$ at each $K$ value is mapped into a fixed $E$ grid. After the diffusion in $K$ is performed, $\bar{f}_{s}$ is mapped back into $M$ grids by interpolation and then other processes, such as drifts, charge exchange, and Coulomb losses are performed. The resulting PADs with this additional diffusion process for 100 $154 \mathrm{keV}$ ions are shown by dashed curves in the bottom panels of Figure 4. In this case, $D_{\alpha \alpha}=5 \times 10^{-6} \mathrm{~s}^{-1}$ provided a good match to the data.

Next we estimate the wave intensity corresponding to the diffusion coefficient obtained above. Assuming a pure pitch angle diffusion, the wave frequency is much less than the gyrofrequency of the ring current ions. In the cold plasma theory, the diffusion coefficient can be roughly approximated as [Cornwall et al., 1970]

$$
D_{\alpha \alpha} \sim \omega_{g}\left(\frac{\delta B}{B}\right)^{2}
$$

where $\omega_{g}$ is the ion gyrofrequency and $\delta B$ is the total wave amplitude. For $100 \mathrm{keV} \mathrm{H}^{+}$at $L=5$, a $D_{\alpha \alpha}$ of $5 \times 10^{-6} \mathrm{~s}^{-1}$ gives $\delta B \sim 0.1 \mathrm{nT}$. This amplitude of magnetic field fluctuation is consistent with the low-frequency wave observations by the AMPTE [LaBelle et al., 1988; Anderson et al., 1992] and the GEOS satellites [Roux et al., 1982] and corresponds to a moderate ion cyclotron fluctuation.

\section{Induced Electric Field}

It is possible to obtain a qualitatively realistic ring current from a simple global enhancement of the magnetospheric convection electric field [Chen et al., 1994]. However, it is well known that the nightside magnetic field undergoes a series of stretching and relaxation cycles, each associated with isolated substorm events [e.g., Moore et al., 1981], that each of these cycles is associated with a "convection surge" [Quinn and Southwood, 1982], and that particle acceleration associated with these surges contributes in some way to magnetic storm periods [Mauk and Meng, 1983]. The induced electric field associated with these events is not global but rather localized in the evening sector. Lyons and Williams [1980] argued that this localization of the injection fields is quantitatively critical to the formation of the ring current. Chen et al. [1994] constructed the substorm-associated convection surges by a superposition of randomly occurring impulses that rise sharply and decay exponentially with a lifetime of $20 \mathrm{~min}$, but treated these as variations of the crosstail potential drop or, equivalently, the global convection strength.

In an attempt to incorporate the localized character of substorm-induced electric fields, we have adopted an approach that is grounded in observations of plasma dynamics at geosynchronous orbit, which corresponds well with the outer boundary of our simulation space. To a steady global convection pattern, we add the drift velocity $(\mathbf{E} \times \mathbf{B})$ induced by changes in a model magnetic field, using an approach that is similar to that used by Delcourt et al. [1990] to describe variations of the fields in the more distant tail. That is, the Tsyganenko magnetic field model (in this case, Tsyganenko [1989] EXT89AE routine) is given a time dependence through the observed variations of the $A E$ index, plotted at 1 -min time resolution in Figure 5 for the day of May 2, 1986. The magnetic field configuration is associated with six levels of the $A E$ index, as shown in Table 1 . Our procedure honors this association of the Tsyganenko level with $A E$, while introducing large variations around the average at moderate activity levels, as follows.

The Tsyganenko level $(T L)$ is initially set at a level, $T L_{i}$, that could be determined by knowledge of the instantaneous geotail configuration, if known, but is treated as a free parameter. $T L$ is assumed to closely track $A E$ at low values of $A E$, according to the Tsyganenko relationship. However, it is taken to grow linearly at a rate proportional to the excess of $A E$ over a critical value, $A E_{\mathrm{o}}$. The growth rate tracks $A E$ in such a way that the induced flow increases with $A E$. To simulate collapse of the tail owing to an instability, $T L$ abruptly decreases immediately upon reaching a threshold value of $T L_{\max }$ (generally set equal to 6 , the maximum $T L$ available). The amplitude of the decline depends on the $A E$ value at the beginning of the field line collapse. However, the minimum duration of the expansion phase is set to be $2 \mathrm{~min}$. After each collapse or expansion phase, $T L$ then resumes tracking the $A E$ index as before. During the growth phase, earthward convection in the tail is suppressed by this procedure, while during the expansion phase it is dramatically increased. The average rate of convection increases with $A E$ at low levels, while tracking it closely. At higher levels, convection undergoes cycles of suppression and enhancement, but the average convection rate must ultimately match that of the underlying convection, a basic constraint that this procedure preserves.

This algorithm for modeling the induced electric field as a series of substorms is summarized mathematically in the following:

Initial level (free)

$$
T L=T L_{i}
$$

Growth or recovery

$$
\frac{d T L}{d t}=C_{1}\left(A E-A E_{\mathrm{o}}\right)
$$

$$
\begin{aligned}
& \text { Expansion } \\
& \qquad \frac{d T L}{d t}=-C_{2} \quad \text { duration }=\max \left[2 \mathrm{~min}, \frac{2\left(6-T L_{e}\right)}{C_{2}}\right]
\end{aligned}
$$

where $A E_{0}=25 \gamma, C_{1}=2 \times 10^{-6} \mathrm{~s}^{-1} \gamma^{-1}, C_{2}=0.25 \mathrm{~min}^{-1}$, and $T L_{e}$ is the Tsyganenko level according to Table 1 at the beginning of the expansion. $C_{1}$ is chosen in a way that the ion drift on the nightside in response to the field line stretching will not exceed the earthward convective drift to prevent net tailward motion on the nightside. $C_{2}$ is chosen such that the complete dipolarization from level 6 to 1 takes place in $20 \mathrm{~min}$. The change in Tsyganenko level during dipolarization is controlled by the duration in such a way that $T L_{e}$ is at the middle of $T L_{\max }$ and the bottom level at the end of the dipolarization. However, during very active periods that whenever $T L_{e} \geq 5.75$, the duration of dipolarization is set to be $2 \mathrm{~min}$ and $T L$ drops from level 6 to 5.5 .

Following the above algorithm and with $T L_{i}$ set to be 5.5 , the simulated Tsyganenko level as a function of time during 

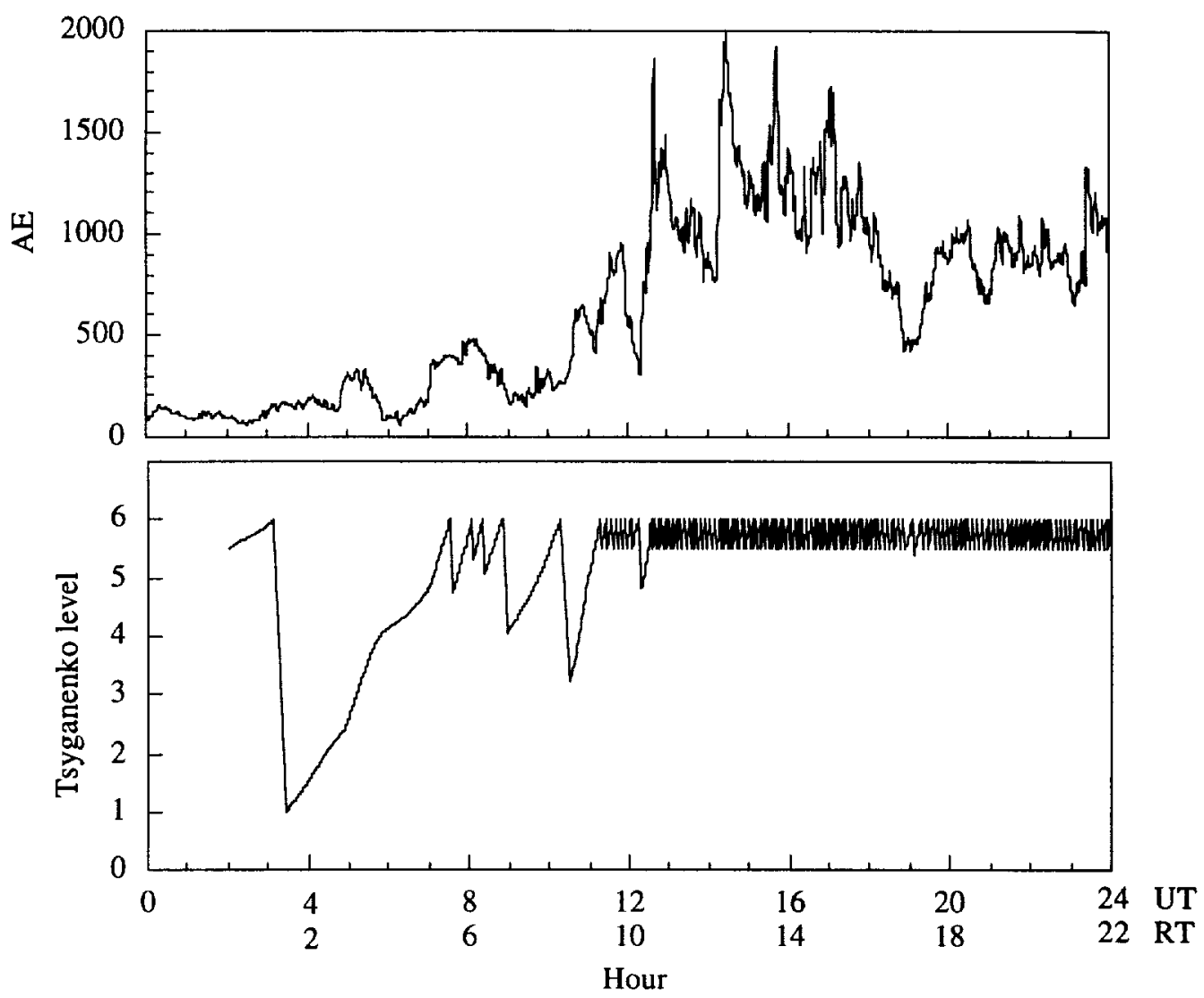

Figure 5. (top) 1-min $A E$ index on May 2, 1986. (bottom) Simulated instantaneous magnetic configuration represented by levels of the Tsyganenko model.

the main phase of the storm is plotted in Figure 5 together with the $A E$ index. As shown in Figure 5, there are five distinct large-amplitude dipolarization events at about 0320, 0730, 0855, 1025, and 1220 UT. After $1220 \mathrm{UT}$, the geomagnetic activity is so strong that repeated rapid stretching and collapsing of the magnetic field occurs accompanied by only small-amplitude changes in $T L$. The predicted substorms qualitatively resemble the injections of energetic electrons observed by the Los Alamos National Laboratory (LANL) geosynchronous satellites. LANL data for May 2, 1986, show clear particle injections at about $0300,0700,1030$, and 1220 UT, and injection cycles are nearly continuous after $1220 \mathrm{UT}$ (G. Reeves, unpublished data, 1995). Although the model does not exactly reproduce the number or timing of injections reported by the LANL satellites, it duplicates the important features of isolated substorms before $1220 \mathrm{UT}$ and multiple substorms in rapid succession thereafter. The free parameter $T L_{i}$ was chosen to obtain good agreement in time between the predicted and the observed discrete injections.

The most direct approach to calculating the drift of ions as a result of the changing magnetic field might be to calculate the induced electric field by "uncurling" $\partial \mathbf{B} / \partial t$ and then calculate the corresponding $\mathbf{E} \times \mathbf{B}$ drift. However, when using an empirical magnetic field model, it is considerably more straightforward to apply Alfven's theorem, also known as the frozen-in field theorem. For purposes of computing the induced field motion, each field line can be regarded as fixed and rooted in the ionosphere, since magnetic field variations are vanishingly small there. The $\mathbf{E} \times \mathbf{B}$ drift velocity of an ion at any point along a field line is equivalent to the perpendicular velocity of that field line at that particular position [Wolf, 1983]. Therefore the induced equatorial drift velocity is calculated from the instantaneous velocity of the equatorial crossing of magnetic field lines in response to the temporal variation of magnetic level shown in Figure 5. This substorminduced drift is added to the gradient curvature drift and the convection electric drift to move the ions. Though the motions of the Tsyganenko magnetic field lines during substorms are considered in calculating the induced drifts, a constant dipole field is assumed in the rest of the calculations. This is a rough and non-self-consistent way of implementing the time-varying magnetic field, which is adequate only in the inner magnetosphere. A more self-consistent treatment will be pursued in future work.

Table 1. $A E$ parameterization of Tsyganenko Magnetic Field Level

\begin{tabular}{lc}
\hline Level & $A E$ \\
\hline 1 & $0-50$ \\
2 & $50-100$ \\
3 & $100-150$ \\
4 & $150-250$ \\
5 & $250-400$ \\
6 & 400 and up \\
\hline
\end{tabular}


(a) quiescent radial convection at $\mathrm{Kp}=7$

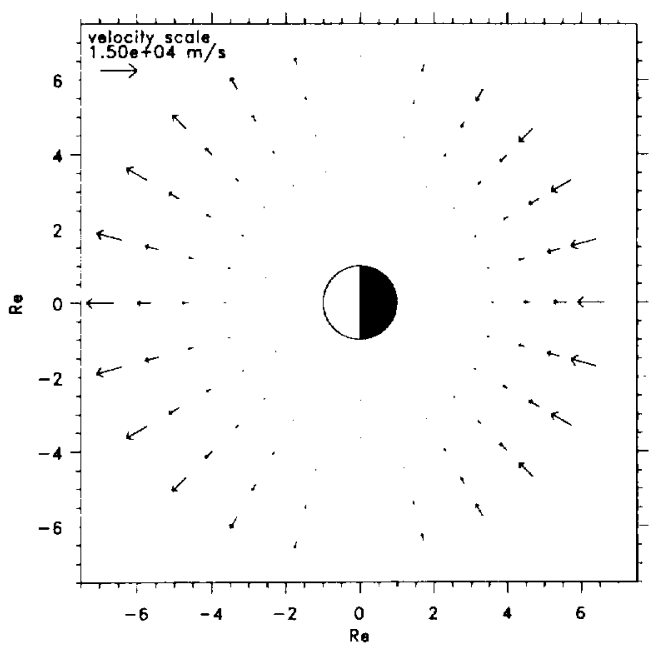

(b) induced radial drift during dipolarization

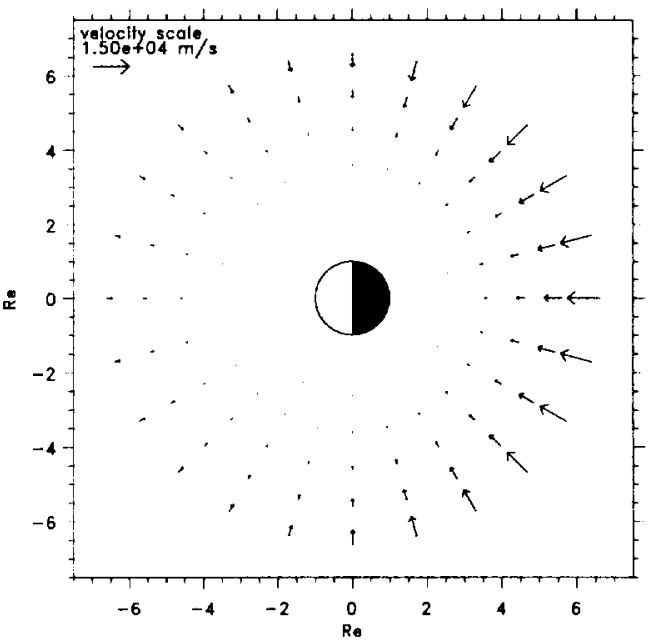

Figure 6. Radial components of (a) steady convection drift velocity at $K p=7$ and (b) induced velocity during dipolarization.

Figure 6 compares the radial component of quiescent convective drift at $K p=7$ with that of the induced velocity during dipolarization. The corresponding azimuthal electric field that cause these drifts can be estimated by $E_{\phi}=B \cdot v_{\text {radial }}$. In this case, the induced electric field at midnight at $L=6$ during dipolarization is about $1.3 \mathrm{mV} / \mathrm{m}$, which is comparable with the quiescent convection field at the same location. However, the inductive electric field is more localized, having $E_{\phi} \propto L^{k}$, where $k \sim 1.2$, compared with $k=1$ in the quiescent field model. As shown in Figure 6, the magnitude of the steady convection is day-night symmetric (Figure 6a). Ions with open drift paths moving in from the tail will drift out the dayside magnetopause with the same speed. Most of the energy gained by particle injection and energization on the nightside is lost by particle drift out at the dayside magnetopause [Kozyra et al., 1994]. However, during the main phase of a storm, the steady intensification of convection leads to deeper penetration of ions (Plate 1a) and increase of particle energy. With the consideration of changing magnetic field, the drift due to the induced electric field is stronger on the nightside than on the dayside during the expansion of a substorm (Figure $6 \mathrm{~b}$ ). The large resultant velocity of convection and dipolarization on the nightside pushes ions close to the Earth before they are trapped [Lyons and Williams, 1980]. As a result, the energy content in the ring current has a sudden jump during the dipolarization event. The situation during the field line stretching is the opposite. The induced radial drifts during stretching are in the opposite directions of those shown in Figure $6 \mathrm{~b}$, and with smaller magnitudes. The tailward motion of field lines counter balances the steady earthward convection on the nightside. The drift loss at the dayside magnetopause thus exceeds the input from convective energization. The energy content in the ring current may drop during the substorm growth phase.

Figure 7 shows the $\mathrm{H}^{+}$energy as a function of simulation time with and without the inclusion of the induced electric field. As shown in the figure, the quiescent convection causes a steady growth in the $\mathrm{H}^{+}$energy (solid curve). In the case of an additional inductive drift, the $\mathrm{H}^{+}$energy fluctuates as field lines undergo stretching and collapsing. During the active period of 10 to $22 \mathrm{RT}$, the $\mathrm{H}^{+}$energy oscillates rapidly about a mean energy that is a bit less than the energy obtained by steady convection only. It was initially very surprising to us that less ring current is produced when storage-release cycles of localized inductive convection are superposed upon a steady convective flow. Upon closer examination of the results, we see that this is true only for the early main phase, when convection is weak so that large cycles of growth and

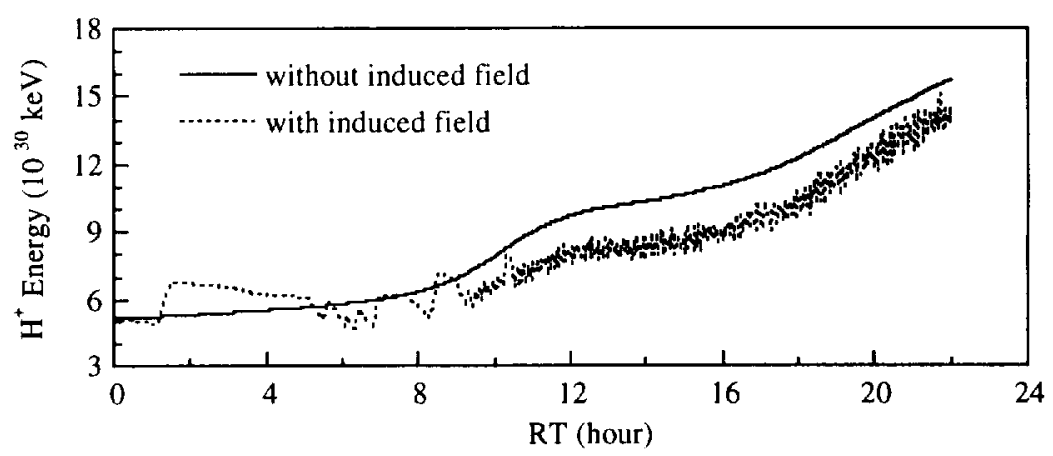

Figure $7 . \mathrm{H}^{+}$energy as a function of simulation time during the storm main phase with and without the consideration of the induced electric field. 
dipolarization occur. During that phase, inclusion of the inductive fields clearly fails to produce as much ring current as the steady case with the same mean convection. However, during the later phase, when convection has increased so that growth is as fast as dipolarization, we see that the two cases in Figure 7 track each other closely.

One interpretation of this is that localized sporadic injection is not an efficient process for creating a full ring current. At low storm power levels, in the absence of a strong steady convection, the freshly injected particles are not able to form a symmetric ring current. As a result, the bursty increase of the ring current during dipolarization is more than balanced by the reduced convection during the slow and long (on the order of hours) growth phase. At higher storm power levels, the cycles of growth and dipolarization are small-amplitude perturbations on the fast global convection that has become the dominant process. The high power levels smooth out the response of the magnetotail, which spends most of its time in more highly stretched states, and transmits energy into the ring current relatively directly and without significant storage and release.

The large amplitudes of the substorm-associated fluctuations in the ion energy content shown in Figure 7 are not supported by the $\mathrm{H}$ component of the high time resolution $(1 \mathrm{~min}$ ) symmetric disturbance index (SYM-H) [Iyemori, 1990], which, on May 2, 1986, fluctuated with amplitudes smaller than those calculated (T. Iyemori, unpublished data, 1995). However, direct inference of ring current energy content from the surface field perturbation requires steady configurations of the magnetic field and the trapped particles [Sckopke, 1966]. A more accurate way to predict the magnetic perturbation is to calculate the current density produced by the energetic ions. It was found that the pressure gradient is the main contribution to the current density [Lui et al, , 1987], and the effect is scaled inversely by $B$. During substorm injections, particles are transported earthward and experience a stronger magnetic field. The increase in the current density produced by these injected particles may somehow be reduced by the larger local $B$ values. We expect the field depression produced by the ring current to fluctuate with a smaller amplitude than the energy content does during substorms when the self-consistently time-dependent magnetic field is taken into account in the calculation.

\section{Discussion and Summary}

The evolution of the ring current ions from an initially quiet time distribution has been addressed as a response to the storm time enhanced convection field and particle injection. Although simple models for the magnetic and electric field are used, our model reproduces the major features of the storm time ring current, such as the day-night asymmetry of ion distributions and deep penetration of ring current ions at the peak of the storm. Under the assumption of a magnetic dipole field, there are simple analytical expressions for the bounceaveraged drift velocities [Ejiri, 1978]. Others have calculated the drift motion in a nondipole field [Schulz and Chen, 1995; Chan et al., 1995].

We have introduced a scheme for solving pitch angle diffusion in an $M-K$ space. Phase space densities are expressed in terms of adiabatic variables $M$ and $K$ in solving drifts and collisional losses, while $E-K$ parameterization is used for the pitch angle diffusion. If an $M-K$ parameterization is used throughout the calculation, a pure pitch angle diffusion may result in diffusion in $M$, diffusion in $K$, and terms corresponding to mixed diffusion of $M$ and $K$. The advantage of switching between two domains is that one can have a simple expression for the left-hand side of the kinetic equation (1), without terms of $\dot{M}$ and $\dot{K}$, and can simultaneously avoid dealing with mixed diffusion on the right-hand side of the equation.

The effect of the stretching and collapsing of the magnetosphere is represented by an additional drift velocity induced by this field line motion. As a consequence, the energy content in the ring current oscillates about a mean value, which does not change significantly because of the substorminduced activities but grows steadily as the convection electric field is enhanced. This result supports the two-step process of ring current formation: during dipolarization events, the earthward transport of plasma sheet population forms a spatially sharp boundary of energetic plasma near the geosynchronous orbit [Mauk and Meng, 1987], then particles undergo further earthward displacement and are energized adiabatically by the convection electric field. The time scale of substorm activities is on the order of $10 \mathrm{~min}$ or less, which is shorter than or comparable to the bounce periods of lowenergy ions ( $<1 \mathrm{keV})$ at $L>5$. Under this condition, the assumption of conserved $J$ (or $K$ ) and the concept of bounce averaging are no longer valid. The lower limit of the particle energy has to be high enough to ensure the conservation of the second adiabatic invariant.

In summary, we have used a 3-D ring current model to simulate the main phase of a magnetic storm similar to that on May 2, 1986. Modeling results are compared with the AMPTE/CCE particles measurements. We found

1. The calculated average $\mathrm{H}^{+}$fluxes agree well with measurements, except the model cannot reproduce the observed ion fluxes at energies below tens of $\mathrm{keV}$ at $L<2.5$.

2. Near the inner edge of the ring current, ion flux peaks at $90^{\circ}$ pitch angle, as a result of strong charge exchange loss of the field-aligned ions.

3. For $\mathrm{H}^{+}$energies greater than tens of $\mathrm{keV}$, the round shape of the pitch angle distribution cannot be solely explained by the charge exchange process and the drift motion. Diffusion in pitch angle due to interactions with ion cyclotron wave are suggested to be responsible for the observed distribution. A pitch angle diffusion process with diffusion coefficient about $5 \times 10^{-6} \mathrm{~s}^{-1}$ is required in order to match the data and is consistent with observed fluctuation amplitudes.

4. The energy content in the ring current fluctuates in response to the field line stre:ching and collapsing during

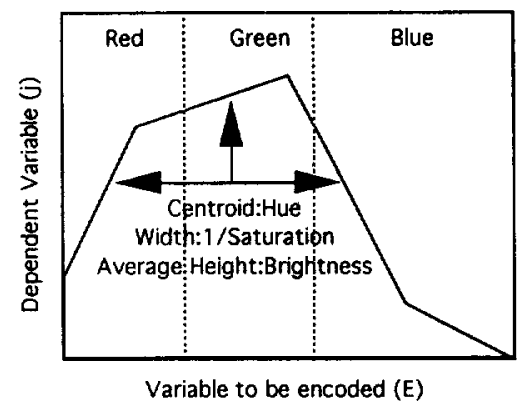

Figure A1. Curve $j(E)$ is divided into three subranges that are associated with red, green, and blue light. 
Table A1. Guide to the Interpretation of Chromogram Color

\begin{tabular}{ll}
\hline Color & \multicolumn{1}{c}{ Interpretation } \\
\hline Gray & no dependence on $E$ (flat) \\
Red & strongly peaked at low values of $E$ \\
Green & strongly peaked at midscale in $E$ \\
Blue & strongly peaked at high values of $E$ \\
Yellow & weakly peaked toward low $E$ \\
Cyan & weakly peaked toward high $E$ \\
Magenta & peaked at high and low $E$ \\
\hline
\end{tabular}

substorms. However, the overall increase in the ring current energy is a result of the gradual enhanced convection field.

\section{Appendix: Description of a Chromogram}

The goal of a chromogram is to plot a function of three variables, $j(L, \phi, E)$, in a two-dimensional color plot. The dependence on the first two variables (i.e., $L$ and $\phi$ ) forms a pixel image in a two-dimensional $L-\phi$ space, while the dependence on the third variable $(E)$ is encoded as color. The encoding of color uses the concept of three basic colors (photon energies) of light: red, green, and blue. Variable $E$ is divided into three ranges (Figure $A 1$ ), associated with red (R), green $(\mathrm{G})$, and blue (B), respectively. The average values of $j$ in these ranges are used to scale the $R, G, B$ components of the encoded color. The hue of the resulting color corresponds to the centroid or mean $E$ of $j(E)$. The saturation of the resulting color corresponds inversely to the width of $j(E)$. Saturation measures the degree of dominance of one hue over the others. As color is varied from gray to a pure hue (from the center to the perimeter of a color wheel shown in Plate 1), saturation varies from 0 to $100 \%$ at constant brightness. The brightness of the encoded color corresponds to the average $j$ over the full range of $E$. Table Al summarizes the interpretation of the color code. The rough energy distribution at each $L-\phi$ location can be inferred from the color at that particular pixel. The detailed features of energy spectra cannot be resolved using the chromogram representation, but it is useful to indicate the spatial dependence of the energy distribution.

Acknowledgments. The authors wish to thank George Khazanov, Doug Hamilton, and Janet Kozyra for many valuable comments and discussions. The 1-minute $A E$ index of May 1986 is provided by Wesley Morris of the National Geophysical Data Center. This work was supported in part by the NASA Space Physics Division under grant UPN 432-20-00. Support at the University of Maryland was from NASA grant NAG5-716. One of us, M.-C. Fok, held a National Research CouncilMarshall Space Flight Center Research Associateship while this work was performed.

The Editor thanks two referees for their assistance in evaluating this paper.

\section{References}

Anderson, B. J., R. E. Erlandson, and L. J. Zanetti, A statistical study of Pc 1-2 magnetic pulsations in the equatorial magnetosphere, 1 , Equatorial occurrence distributions, J. Geophys. Res., 97, 3075-3088, 1992.

Borovsky, J. E., M. F. Thomsen, and D. J. McComas, The ion and electron plasma sheets at geosynchronous orbit: A statistical overview, Eos Trans. AGU, 75(44), Fall Meet. Suppl., 576, 1994.
Chan, A. A., K. E. Braaten, and R. A. Wolf, Relativistic bounceaveraged drift equations for radiation belt modeling with the MSFM, Eos Trans. AGU, 76(17), Spring Meet. Suppl., S254, 1995.

Chen, M. W., M. Schulz, L. R. Lyons, and D. J. Gorney, Stormtime transport of ring current and radiation belt ions, J. Geophys. Res., 98, 3835-3849, 1993.

Chen, M. W., M. Schulz, and L. R. Lyons, Simulations of phase space distributions of stormtime proton ring current, J. Geophys. Res., 99, 5745-5759, 1994.

Cornwall, J. M., F. V. Coroniti, and R. M. Thorne, Turbulent loss of ring current protons, J. Geophys. Res., 75, 4699-4709, 1970.

Davidson, G. T., An improved empirical description of the bounce motion of trapped particles, J. Geophys. Res., 8I, 4029-4030, 1976.

Delcourt, D. C., J. A. Sauvaud, and A. Pedersen, Dynamics of single particle orbits during substorm expansion phase, J. Geophys. Res., 95, 20,853-20,865, 1990.

Ejiri, M., Trajectory traces of charged particles in the magnetosphere, $J$. Geophys. Res., 83, 4798-4810, 1978.

Fok, M.-C., J. U. Kozyra, A. F. Nagy, C. E. Rasmussen, and G. V. Khazanov, Decay of equatorial ring current ions and associated aeronomical consequences, J. Geophys. Res., 98, 19,381-19,393, 1993.

Fok, M.-C., T. E. Moore, J. U. Kozyra, G. C. Ho, and D. C. Hamilton, Three-dimensional ring current decay model, J. Geophys. Res., 100, 9619-9632, 1995a.

Fok, M.-C., P. D. Craven, T. E. Moore, and P. G. Richards, Ring current - plasmasphere coupling through Coulomb collisions, in Cross-Scale Coupling in Space Plasmas, Geophys. Monogr. Ser., vol, 93, edited by J. L. Horwitz, N. Singh, and J. L. Burch, pp. 161-171, AGU, Washington, D. C., 1995b.

Harel, M. R. A. Wolf, P. H. Reiff, R. W. Spiro, W. J. Burke, F. J. Rich, and $M$. Smiddy, Quantitative simulation of a magnetospheric substorm, 1, Model logic and overview, J. Geophys. Res., 86, 2217$2241,1981$.

Horwitz, J. L., The ionosphere as a source of magnetospheric ions, Rev. Geophys., 20, 929-952, 1982.

lyemori, T., Storm-time magnetospheric currents inferred from midlatitude geomagnetic field variations, J. Geomagn. Geoelectr., 42, 1249-1265, 1990.

Kennel, C. F., and H. E. Petschek, Limit on stably trapped particle fluxes, J. Geophys. Res., 7I, 1-28, 1966

Kistler, L. M., F. M. Ipavich, D. C. Hamilton, G. Gloeckler, B. Wilken, G. Kremser, and W. Studemann, Energy spectra of the major ion species in the ring current during geomagnetic storms, J. Geophys. Res., 94, 3579-3599, 1989.

Kozyra, J. U., V. K. Jordanova, M.-C. Fok, and C. R. Clauer, Energy storage and release in the Earth's ring current, Eos Trans. AGU, 75(44), Fall Meet. Suppl., 551, 1994.

LaBelle, J., R. A. Treumann, W. Baumjohann, G. Haerendel, N. Sckopke, G. Paschmann, and H. Lühr, The duskside plasmapause/ ring current interface: Convection and plasma wave observations, $J$. Geophys. Res., 93, 2573-2590, 1988.

Lui, A. T. Y., R. W. McEntire, and S. M. Krimigis, Evolution of the ring current during two geomagnetic storms, $J$. Geophys. Res., 92, 74597470,1987

Lyons, L. R., and D. J. Williams, A source for the geomagnetic storm main phase ring current, J. Geophys. Res., 85, 523-530, 1980.

Mauk, B. H., Quantitative modeling of the "convection surge" mechanism of ion acceleration, J. Geophys. Res., 91, 13,423-13,431, 1986.

Mauk, B. H., and C.-l. Meng, Characterization of geostationary particle signatures based on the 'injection boundary' model, J. Geophys. Res., $86,3055-3071,1983$.

Mauk, B. H., and C.-I. Meng, Plasma injection during substorms, Phys. Scr. T., 18, 128-139, 1987.

Maynard, N. C., and A. J. Chen, Isolated cold plasma regions: Observations and their relation to possible production mechanisms, $J$. Geophys. Res., 80, 1009-1013, 1975. 
Mcllwain, C. E., Magnetic coordinates, Space Sci. Rev., 5, 585-598, 1966.

Mcllwain, C. E., Plasma Convection in the vicinity of the geosynchronous orbit, in Earth's Magnetospheric Processes, edited by B. M. McCormac, pp. 268-279, D. Reidel, Norwell, Mass., 1972.

Moore, T. E., R. L. Arnoldy, J. Feynman, and D. A. Hardy, Propagating substorm injection fronts, J. Geophys. Res., 86, 6713-6726, 1981.

Quinn, J. M., and D. J. Southwood, Observations of parallel ion energization in the equatorial region, $J$. Geophys. Res., 87, 10,536$10,540,1982$.

Roederer, J. G., Dynamics of Geomagnetically Trapped Radiation, Springer-Verlag, New York, 1970.

Roux, A., S. Perraut, J. L. Rauch, C. de Villedary, G. Kremser, A. Korth, and D. T. Young, Wave-particle interactions near $\Omega_{\mathrm{He}^{+}}$observed on board GEOS 1 and 2,2, Generation of ion cyclotron waves and heating of $\mathrm{He}^{+}$ions, J. Geophys. Res., 87, 8174-8190, 1982.

Schulz, M., and M. W. Chen, Bounce-averaged Hamiltonian for charged particles in an axisymmetric but nondipolar model magnetosphere, J. Geophys. Res., 100, 5627-5635, 1995.

Sckopke, N., A general relation between the energy of trapped particles and the distribution field near the Earth, J. Geophys. Res., 71, 3125$3130,1966$.

Sheldon, R. B., and D. C. Hamilton, Ion transport and loss in the Earth's quiet ring current, 1, Data and standard model, J. Geophys. Res., 98, 13,491-13,508, 1993

Stern, D. P., The motion of a proton in the equatorial magnetosphere, $J$. Geophys. Res., 80, 595-599, 1975.

Tsyganenko, N. A., A magnetospheric magnetic field model with a warped tail plasma sheet, Planet. Space Sci., 37, 5-20, 1989.
Volland, H., A semiempirical model of large-scale magnetospheric electric fields, J. Geophys. Res., 78, 171-180, 1973.

Williams, D. J., and L. R. Lyons, The proton ring current and its interaction with the plasmapause: Storm recovery phase, $J$. Geophys. Res., 79, 4195-4207, 1974.

Williams, D. J., and M. Sugiura, The AMPTE Charge Composition Explorer and the 4-7 September 1984 geomagnetic storm, Geophys. Res. Lett., 12, 305-308, 1985.

Wolf, R. A., The quasi-static (slow-flow) region of the magnetosphere, in Solar-Terrestrial Physics, edited by R. L. Carovillano and J. M. Forbes, D. Reidel, Norwell, Mass., 1983.

Wolf, R. A., M. Harel, R. W. Spiro, G.-H. Voigt, P. H. Reiff, and C.-K. Chen, Computer simulation of inner magnetospheric dynamics for the magnetic storm of July 29, 1977, J. Geophys. Res., 87, 5949-5962, 1982.

M.-C. Fok, Uni . *rsities Space Research Association, Space Sciences Laboratory, NASA Marshall Space Flight Center, Huntsville, AL 35812. (e-mail: fok@msfc.nasa.gov)

M. E. Greenspan, Department of Physics, University of Maryland, College Park, MD 20742. (e-mail: greenspan @umdsp.umd.edu)

T. E. Moore, Space Sciences Laboratory, NASA Marshall Space Flight Center, Huntsville, AL 35812. (e-mail: t.e.moore@msfc.nasa.gov)

(Received December 15, 1995; revised April 19, 1996; accepted April 19, 1996.) 